\title{
Avaliação dos diferentes protocolos de tratamento da Disfunção Temporomandibular miogênica: Revisão de Literatura
}

\author{
Evaluation of the different protocols for the treatment of \\ Temporomandibular Disorders myogenic: Literature Review
}

Iara Roberta Alves Rodrigues ${ }^{1}$

luana Cardoso Cabral ${ }^{2}$

LIVIA BONJARDIM LIMA ${ }^{2}$

Paulo Cézar Simamoto JúnioR ${ }^{3}$

Alfredo Júlio Fernandes Neto ${ }^{3}$

Marlete RibeiRo da Silva ${ }^{4}$

\begin{abstract}
RESUMO
Introdução: A disfunção temporomandibular (DTM) miogênica é considerada uma alteração musculoesquelética do sistema mastigatório. Além disso, é caracterizada por distúrbios que afetam a amplitude do movimento mandibular e articular. Devido ao carácter multifatorial, vários tratamentos, isolados ou concomitantes, são indicados para essa disfunção. Apesar de diversos estudos disponíveis na literatura sobre essas modalidades de terapia, esses são pouco conclusivos.

Objetivo: Revisar a literatura apresentando os diferentes protocolos de tratamento empregados na DTM miogênica.

Materiais e Métodos: A estratégia de busca foi realizada na base de dados do Pubmed, Scielo e Cochrane por meio dos seguintes descritores: Temporomandibular Joint Disorders, Myofascial Pain Syndromes, treatment, Randomized Controlled Trial. A avaliação dos estudos foi efetuada em duas etapas por revisores independentes. Na primeira etapa, os títulos e resumos foram investigados e aqueles que apresentassem os critérios de elegibilidade eram selecionados. Na segunda etapa, os artigos foram lidos na íntegra e posteriormente incluídos conforme o consenso dos dois revisores baseando-se nos critérios de inclusão e exclusão.
\end{abstract}

Resultados: 100 citações foram identificadas e 43 lidas na íntegra. Oito artigos reuniram os critérios de elegibilidade, contudo dois foram excluídos devido à baixa qualidade da evidência. Diversos tratamentos foram abordados como orientações ao paciente, tratamento fisioterapêutico, odontológico e terapia medicamentosa.

Conclusão: As terapias como as orientações, treinamento com relaxamento muscular, fármacos, placa estabilizadora da oclusão e acupuntura são eficazes no controle da DTM miogênica.

Palavras-chave: Síndrome da Disfunção da Articulação Temporomandibular. Mialgia. Estudos randomizados.

\begin{abstract}
Introduction: Myogenic temporomandibular disorder (TMD) is a musculoskeletal variation of the masticatory system. In addition, it is characterized by disorders that affect the range of mandibular and joint movement. Because of the multifactorial nature, several treatments, isolated or concomitant, are indicated for this dysfunction. Although several studies available in the literature on these modalities of therapy, these are little conclusive. Objective: To review the literature presenting the different treatment protocols used in myogenic TMD.
\end{abstract}

Cirurgiã-dentista pela Universidade Federal de Uberlândia - Uberlândia - MG, Brasil.

Doutoranda em Odontologia na Universidade Federal de Uberlândia - Uberlândia - MG, Brasil.

3 Prof. Dr. da área de oclusão, prótese fixa e materiais odontológicos da Faculdade de Odontologia da Universidade Federal de Uberlândia - Uberlândia - MG, Brasil.

4 Profa. Dra. da área de oclusão, prótese fixa e materiais odontológicos da Faculdade de Odontologia da Universidade Federal de Uberlândia - Uberlândia - MG, Brasil. 


\begin{abstract}
Materials and Methods: The search strategy was performed using the following descriptors: Temporomandibular Joint Disorders, Myofascial Pain Syndromes, treatment, Randomized Controlled Trial, Pubmed, Scielo and Cochrane. The studies were evaluated in two stages by independent reviewers. In the first step, the titles and abstracts were investigated and those that presented the eligibility criteria were selected. In the second stage, the articles were read in full and subsequently included according to the consensus of the two reviewers based on the inclusion and exclusion criteria.

Results: 100 citations were identified and 43 citations were read in full. Eight articles met the eligibility criteria, however two were excluded due to poor quality of evidence. Several treatments were approached as patient orientations, physiotherapeutic treatment, dental treatment and drug therapy.

Conclusion: Therapies such as guidelines, muscle relaxation training, drugs, occlusion stabilizing plate and acupuncture are effective in controlling myogenic TMD.

Key-words: Temporomandibular Joint Dysfunction Syndrome. Myalgia. Randomized Controlled Trial.
\end{abstract}

\section{INTRODUÇÃO}

Deacordo comaAmericanDentalAssociation (ADA), a Disfunção Temporomandibular (DTM) é definida como um grupo de desordens orofaciais, caracterizada por condições que envolvem os músculos da mastigação, as articulações temporomandibulares (ATMs) e estruturas associadas. ${ }^{1}$ A DTM pode ser categorizada como intra ou extracapsular. As desordens intracapsulares estão relacionadas com doenças degenerativas como deslocamento de disco articular, artrite e fibrose. Os distúrbios extracapsulares são principalmente miofasciais, sendo o grupo de DTM mais comum. ${ }^{2}$

A DTM miogênica é considerada uma alteração musculoesquelética do sistema mastigatório. Além disso, é caracterizada por distúrbios que afetam a amplitude do movimento mandibular e articular. ${ }^{3}$ Diversos fatores estão envolvidos na etiologia como os distúrbios oclusais (perdas dentárias, desgaste dental, restaurações inadequadas), psicológicos (a tensão aumenta a atividade muscular causando espasmo e fadiga), hábitos parafuncionais (bruxismo, onicofagia, apoio da mão na mandíbula, sucção digital ou de chupeta), lesões traumáticas ou degenerativas, má postura, fatores estruturais e neuromusculares. ${ }^{4,5,6}$

A dor é o sintoma mais comum dessa desordem e, por ser complexa, requer a avaliação de aspectos biológicos, estruturais, funcionais, emocionais, cognitivos e comportamentais. Embora existam dificuldades para selecionar instrumentos adequados para a averiguação do nível de dor, é importante avaliá-la identificando a gênese, manutenção ou exacerbação, a fim de orientar na decisão dos procedimentos terapêuticos. ${ }^{7}$

A literatura disponível referente às modalidades de tratamento mais adequadas para a DTM muscular apresenta baixa evidência. Estudos clínicos randomizados, acompanhamentos clínicos longitudinais padronizados são escassos e pouco conclusivos. $^{8}$ No entanto, existem várias modalidades de tratamento que podem atuar na redução da sintomatologia. Esses protocolos incluem procedimentos não invasivos como o uso da placa estabilizadora da oclusão, reeducação do paciente por meio do biofeedback, fisioterapia ou procedimentos invasivos como o ajuste oclusal, acupuntura, toxina botulínica e tratamento cirúrgico. ${ }^{9}$

Nos últimos anos, essa desordem tornouse um significante problema de saúde pública, devido à alta prevalência em grupos específicos e por influenciar na qualidade de vida desses indivíduos. ${ }^{10}$ Nesse contexto, o objetivo do estudo foi revisar a literatura apresentando os diferentes protocolos de tratamento empregados na DTM miogênica. 


\section{MATERIAIS E MÉTOdOS}

\section{Critérios de Elegibilidade}

Critérios de Inclusão: Para o levantamento bibliográfico foram incluídos ensaios clínicos randomizados, que avaliaram o tipo de tratamento proposto e a eficácia em indivíduos diagnosticados com DTM de origem muscular. O formato PICOS (problema, intervenção, comparação, desfechos e tipo de estudo) foi adaptado para definir a pergunta clínica:

P - Disfunção temporomandibular de origem muscular.

I - placa estabilizadora da oclusão, biofeedback, acupuntura, terapia psicológica ou farmacológica, ajuste oclusal, fisioterapia, toxina botulínica.

$\mathrm{C}$ - ----------------.

O - avaliação da sintomatologia.

$\mathrm{S}$ - estudos clínicos randomizados.

Critérios de Exclusão: Relatos de caso, série de casos, cartas ao leitor e capítulos de livro foram excluídos da revisão.

\section{Estratégia de busca \\ e seleção dos estudos}

As estratégias de busca foram obtidas por meio do dicionário $\mathrm{DeCs} / \mathrm{MeSH}$ e utilizadas nas bases de dados Pubmed, Scielo e Cochrane com os seguintes descritores ( $\mathrm{MeSH}$ terms): Temporomandibular Joint Disorders, Myofascial Pain Syndromes, treatment, Randomized Controlled Trial.

O estudo foi realizado em duas fases. Na primeira fase, 2 revisores (LCC e IRAR) independentes, avaliaram o título e resumo de todas as citações registradas nas bases de dados. Os estudos que não apresentaram os critérios de inclusão estabelecidos foram descartados. Na fase 2, os artigos selecionados, por meio dos mesmos critérios de elegibilidade, foram avaliados na íntegra (LCC e IRAR). Os dois revisores independentes participaram da fase 2. Nos casos em que houve divergência, um terceiro revisor avaliou o mesmo para o desempate (MRS).

\section{Avaliação da qualidade da evidência}

Os artigos selecionados foram avaliados quanto à qualidade da evidência por meio da Escala Jadad. ${ }^{11}$ Após o preenchimento do quadro, classificou a qualidade dos artigos a partir da soma de $A+B+C+D+E=5$ pontos. Sendo que resultados de $0-2$ pontos foram classificados como baixa qualidade e resultados de 3-5 pontos como alta qualidade. Os escores foram obtidos por meio de: Resposta Sim = $1 \mathrm{e}$ Resposta Não = 0 (Quadro 1).

Aqueles artigos que apresentaram baixa qualidade de evidência foram excluídos.

\begin{tabular}{|l|c|}
\hline \multicolumn{1}{|c|}{ Item } & Escore \\
\hline $\begin{array}{l}\text { A. O estudo foi relatado como } \\
\text { randomizado? }\end{array}$ & $0 / 1$ \\
\hline $\begin{array}{l}\text { B. O esquema de randomização foi } \\
\text { descrito e apropriado? }\end{array}$ & $0 / 1$ \\
\hline $\begin{array}{l}\text { C. O estudo foi descrito como duplo- } \\
\text { cego? }\end{array}$ & $0 / 1$ \\
\hline $\begin{array}{l}\text { D. Foi o método mais adequado de } \\
\text { duplo-cego? }\end{array}$ & $0 / 1$ \\
\hline $\begin{array}{l}\text { D1. Os pacientes foram relatados como } \\
\text { cego? }\end{array}$ & - \\
\hline $\begin{array}{l}\text { D2. O avaliador dos resultados foi } \\
\text { relatado como cego? }\end{array}$ & - \\
\hline $\begin{array}{l}\text { E. Houve descrição das desistências e } \\
\text { retiradas do estudo? }\end{array}$ & $0 / 1$ \\
\hline
\end{tabular}

Quadro 1: Escala Jadad.

\section{Dados registrados}

Dois autores coletaram os dados dos estudos selecionados (LCC e IRAR). As seguintes informações foram registradas: autor, ano, amostra e resultados encontrados. 


\section{Resultados}

$\mathrm{Na}$ pesquisa inicial, 100 artigos foram identificados nas bases de dados. $\mathrm{Na}$ avaliação dos resumos, 57 foram excluídos. Portanto, apenas 43 foram selecionados para a avaliação na fase 2. Desses estudos remanescentes, oito artigos foram incluídos. Após a avaliação da qualidade da evidência, dois estudos foram excluídos, devido ao baixo escore. O fluxograma do processo de identificação, inclusão e exclusão dos estudos é mostrado na Figura 1.

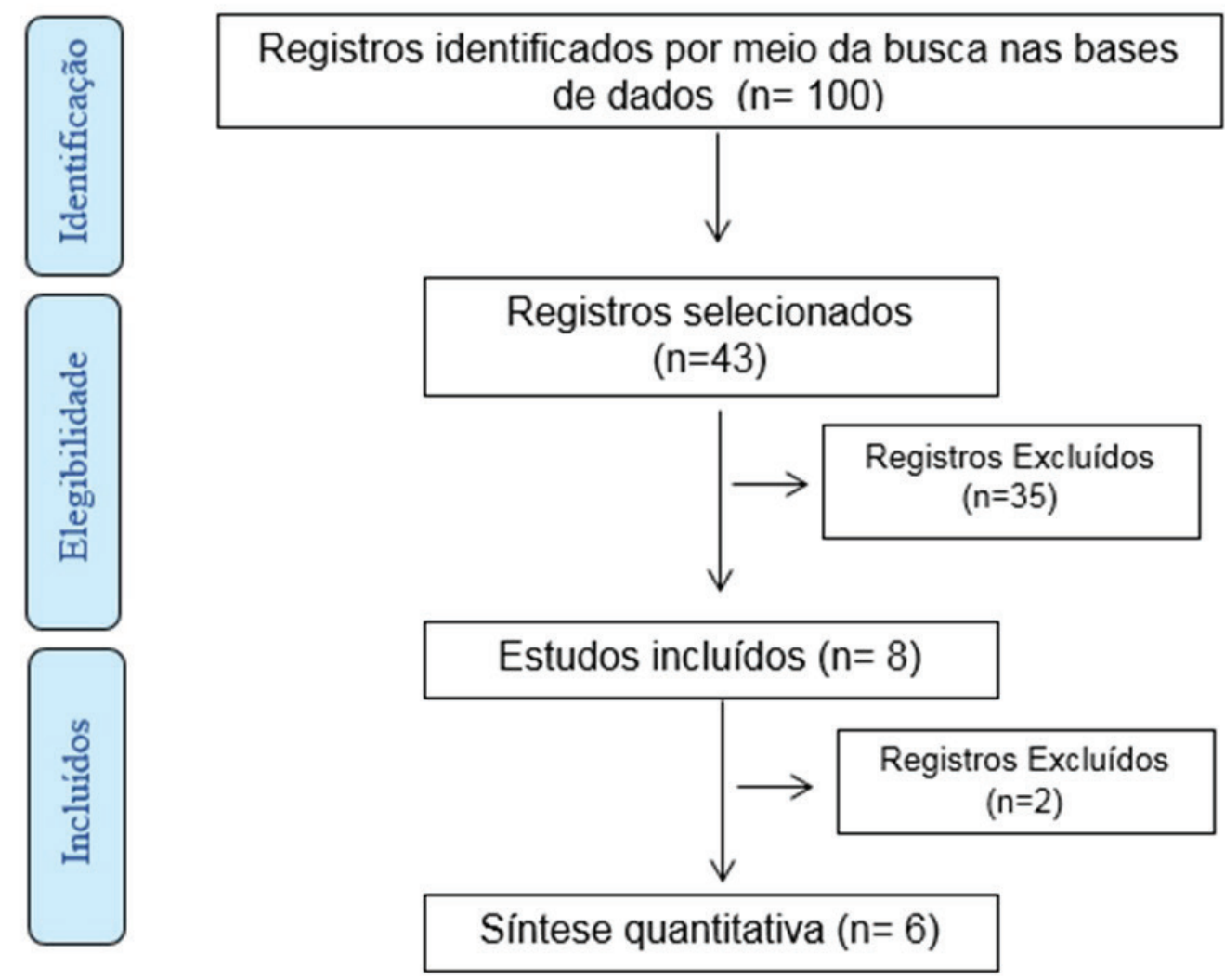

Figura 1. Seleção dos artigos.

A Tabela 1 mostra os artigos e a classificação da qualidade da evidência. Observa-se que seis apresentaram alta qualidade e dois baixa qualidade.

\section{Dıscussão}

Diferentes formas de tratamento têm sido propostas, com o objetivo de reduzir significativamente os sinais e os sintomas da DTM miogênica. O tratamento padrão geralmente consiste de uma combinação de técnicas para melhorar a mobilidade oral, reduzindo a dor musculoesquelética e a inflamação, mesmo que os mecanismos precisos de alguns protocolos sejam desconhecidos. ${ }^{19}$ As intervenções encontradas nessarevisãoincluemorientaçãoeautocuidado, intervenção medicamentosa, treinamento com relaxamento muscular, fisioterapia, uso da placa estabilizadora da oclusão, aparelhos pré-fabricados e acupuntura. 
Tabela 1: Artigos Incluídos.

\begin{tabular}{|c|c|c|c|c|}
\hline Ano & Autores & Amostra & Resultados & Classificação \\
\hline 2015 & Wahlund K. et al. ${ }^{12}$ & $\begin{array}{c}64 \\
\text { pacientes }\end{array}$ & $\begin{array}{c}\text { Terapia com placa estabilizadora da } \\
\text { oclusão foi mais eficaz que terapias de } \\
\text { relaxamento. }\end{array}$ & 5 \\
\hline 2014 & $\begin{array}{c}\text { Alencar Júnior FG } \\
\text { et al. }{ }^{13}\end{array}$ & $\begin{array}{c}45 \\
\text { pacientes }\end{array}$ & $\begin{array}{c}\text { Uso de Tizanidina/Ciclobenza-prina } \\
\text { mais gerenciamento de autocuidado do } \\
\text { paciente não foram mais eficazes que o } \\
\text { placebo. }\end{array}$ & 3 \\
\hline 2012 & Niemela $\mathrm{K}$ et al. ${ }^{14}$ & $\begin{array}{c}80 \\
\text { pacientes }\end{array}$ & $\begin{array}{c}\text { Placa estabilizadora da oclusão, } \\
\text { terapias de autocuidado e exercícios } \\
\text { faciais não foram mais eficazes na dor } \\
\text { miofascial comparado ao autocuidado e } \\
\text { exercícios faciais. }\end{array}$ & 1 \\
\hline 2012 & $\begin{array}{c}\text { Guarda-Nardini L et } \\
\text { al. }^{15}\end{array}$ & $\begin{array}{c}30 \\
\text { pacientes }\end{array}$ & $\begin{array}{l}\text { Injeções de toxina botulínica e técnicas } \\
\text { de manipulação facial melhoraram a } \\
\text { dor miofascial. }\end{array}$ & 2 \\
\hline 2012 & Doepel M et al. ${ }^{16}$ & $\begin{array}{c}75 \\
\text { pacientes }\end{array}$ & $\begin{array}{c}\text { Aparelhos pré-fabricados e placas } \\
\text { estabilizadoras possuem a mesma } \\
\text { eficácia. }\end{array}$ & 5 \\
\hline 2009 & Shen YF et al. ${ }^{17}$ & $\begin{array}{c}28 \\
\text { pacientes }\end{array}$ & $\begin{array}{l}\text { Acupuntura foi eficaz na diminuição } \\
\text { da dor miofascial em comparação ao } \\
\text { placebo. }\end{array}$ & 3 \\
\hline 2009 & $\begin{array}{c}\text { Alencar Junior F, } \\
\text { Becker } A^{7}\end{array}$ & $\begin{array}{c}42 \\
\text { pacientes } \\
\end{array}$ & $\begin{array}{l}3 \text { tipos de placas associadas com } \\
\text { o autocuidado foram eficazes na } \\
\text { diminuição da dor miofascial. } \\
\end{array}$ & 3 \\
\hline 2007 & $\begin{array}{c}\text { Shen YF \& Goddard } \\
\mathrm{G}^{18}\end{array}$ & $\begin{array}{c}15 \\
\text { pacientes }\end{array}$ & $\begin{array}{l}\text { Acupuntura foi eficaz na redução da dor } \\
\text { miofascial em comparação ao placebo. }\end{array}$ & 5 \\
\hline
\end{tabular}

A orientação e autocuidado devem ser incluídos como procedimentos iniciais no plano de tratamento. Essa modalidade objetiva educar o paciente sobre a possível etiologia da desordem, conscientizar quanto à importância da mastigação bilateral simultânea para não sobrecarregar a articulação temporomandibular ou os músculos mastigatórios, orientar sobre o consumo diminuído de cafeína e controle de atividades que contribuem para a hiperatividade muscular como o apertamento dentário. Assim, instruções como a posição de repouso mandibular (dentes levemente separados, lábios ligeiramente fechados, sem pressionar a língua contra os dentes e músculos) devem ser fornecidas aos pacientes. ${ }^{20}$ Além disso, associada às recomendações da educação e autocuidado, a termoterapia, que consiste em aplicações de calor nos músculos masseteres durante 15 minutos até três vezes por dia, é indicada para pacientes com dor orofacial. No estudo de Alencar Junior et al., ao comparar a terapia medicamentosa, autocuidado e placebo, em 45 pacientes diagnosticados com dor miofascial, observaram que não houve diferenças entre os grupos. No entanto, alguns estudos mostram resultados eficazes com essa conduta. Michelotti et al. ${ }^{21}$ encontraram 
que pacientes que receberam educação sobre autogestão demonstraram melhores resultados comparados àqueles que receberam aparelhos oclusais. Similarmente, Dworkin et al. ${ }^{22}$ observaram níveis de dor diminuídos com a implementação do autocuidado no manejo de pacientes com DTM.

Outra modalidade de tratamento é o treinamento do relaxamento muscular por meio de orientações, que incluem instruções escritas e áudio, denominadas de biofeedback. O paciente recebe a instrução de sentarse, concentrando-se principalmente na região superior do corpo, especialmente nos músculos do rosto e pescoço, sem enrijecer e com respiração diafragmática, utilizando uma palavra controlada, por exemplo, "relaxa". É instituído com o intuito de o paciente relaxar pelo menos uma vez por dia durante 15 a 20 minutos. O biofeedback para DTM visa melhorar a propriocepção, conscientização das parafunções orais e reduzir a hiperatividade dos músculos mastigatórios. Os pacientes com DTM apresentam hiperatividade muscular, e a redução desses hábitos, leva à melhora dos sintomas. ${ }^{23}$ Apesar dos benefícios, Wahlund et al. ${ }^{24}$ afirmam que o cumprimento das sessões de relaxamento pelos pacientes durante seis meses de acompanhamento é baixo e que a eficácia do método está diretamente relacionada com os episódios de dor.

Outras condutas para essa desordem são a intervenção por meio dos fármacos. Os agentes comumente usados incluem analgésicos, relaxantes musculares, ansiolíticos e antidepressivos. ${ }^{25}$ A farmacoterapia objetiva diminuir a dor e a inflamação nas ATMs e músculos. Estudos mostram que alguns medicamentos como o uso de Tizanidina ou Ciclobenzaprina isoladamente não são eficazes. ${ }^{13}$ Contudo, pode ser considerada uma terapia complementar. ${ }^{26}$

Ainda sobre tratamentos não invasivos, as sessões múltiplas de manipulação facial fornecem uma pressão digital profunda exercida sobre centros específicos de nódulos musculares selecionados de acordo com exame clínico preciso. Os pacientes são submetidos a três sessões de manipulação facial de 50 minutos por semana. A pressão provoca uma hiperemia que atinge a fáscia profunda do músculo, restaurando as fibras e proporcionando uma melhor adaptação tensional para restabelecer um equilíbrio fisiológico. ${ }^{27}$

A terapia com a placa estabilizadora da oclusão também é um método conservador, ela é confeccionada comumente no arco superior. Ela é utilizada pelo paciente no período noturno ou toda vez que o indivíduo julgar necessário. Esse aparelho oclusal tem sua superfície projetada para ter o máximo de contatos oclusais e guia anterior. ${ }^{27}$ A placa oclusal parece um tratamento clínico eficaz em termos de custos e em termos de tempo de terapia. Ademais, o uso de placas oclusais isoladamente ou em combinação com outras modalidades de tratamento é a forma mais comum de manejo da dor em pacientes com DTM miogênica. ${ }^{28,18}$ Embora a sua eficácia para o alívio da dor parece muitas vezes semelhante a outros tratamentos como a acupuntura, biofeedback e exercícios da mandíbula, ${ }^{29,30,31}$ estudos com adolescentes diagnosticados com dor orofacial apontam efeitos diferenciados favorecendo o tratamento com placa oclusal comparado ao treinamento de relaxamento. ${ }^{24}$

Concomitante a isso, aparelhos préfabricados são confeccionados de resina acrílica e possuem tamanho pequeno comparado às placas estabilizadoras oclusais, já que recobrem somente a superfície de incisivos e caninos com uma extensão palatina de cerca de um centímetro. Entretanto, ambos os aparelhos demonstram ser eficazes no alívio da dor. O aparelho pré-fabricado intraoral parece mais prático, pois é necessário 
apenas uma consulta e não há custos de processamento laboratorial. ${ }^{32}$ Geralmente, esse tipo de aparelho é utilizado como diagnóstico da dor, visto que o uso prolongado pode causar a extrusão dos dentes posteriores.

A acupuntura é outra modalidade de tratamento descrita na literatura. Crescentes evidências suportam o uso no tratamento de condições crônicas. ${ }^{33} \mathrm{~A}$ acupuntura tem efeito analgésico ancorado desde conceitos tradicionais que centram em desequilíbrios de fluxo de energia através do corpo, a teorias ocidentais sobre o papel neural ativado e opioide dos sistemas endógenos. ${ }^{34,35}$ No entanto, é provável que a acupuntura envolva o efeito placebo em pacientes. ${ }^{17,18} \mathrm{~A}$ técnica se baseia no agulhamento com uma profundidade de 10 a 20 milímetros no ponto chamado Hegu LI4, que se localiza no ponto mais alto do músculo adutor entre o polegar e o dedo indicador (o nervo radial inerva essa região). Esse ponto é utilizado para as dores na cabeça e dores de garganta. ${ }^{36}$ Isso sugere que a acupuntura pode aumentar a tolerância funcional do músculo masseter em pacientes com dor miofascial, que têm tolerância de pressão de dor significativamente menor do que pacientes saudáveis. ${ }^{17}$

\section{Considerações finAIS}

As terapias como as orientações, treinamento com relaxamento muscular, fármacos, placa estabilizadora da oclusão e acupuntura são eficazes no controle da DTM miogênica.

\section{REFERÊNCIAS}

1. Carrara SV, Conti PCR, Barbosa JT. Termo do $1^{\circ}$. Congresso em Disfunção Temporomandibular e Dor http:// www.scielo.br/scielo.php?pid=S2176$-94512010000300014 \&$ script $=$ sci_ abstract\&tlng=pt
2. Kraaijenga S., van der Molen L., van Tinteren H., Hilgers F., Smeele L. Treatment of myogenic temporomandibular disorder: a prospective randomized clinical trial, comparing a mechanical stretching device (TheraBite $\AA$ ) with standard physical therapy exercise. Cranio, 2014; 32 (3): 208-16. DOI: 10.1179/0886963413Z.00000000 016.

3. Miller VJ, Karic VV, Myers SL, Exner HV. Myogenous temporomandibular disorder patients and the temporomandibular opening index. $\mathbf{J}$ Oral Rehabil., 2000; 27: 720-2. DOI:10.1046 /j.1365-2842.2000.00562.

4. Xie Q., Li X., Xu X. The difficult relationship between occlusal interferences and temporomandibular disorder - insights from animal and human experimental studies. J Oral Rehabil., 2013; 40 (4): 279-95. DOI: 10.1111/joor.12034.

5. Donnarumma, M. D. C.; Muzilli, C. A.; Ferreira, C.; Nemr, K. Disfunções Temporomandibulares: Sinais, Sintomas e Abordagem Multidisciplinar. Revista Cefac., 2010; 12 (5): 788-94. http:// www.scielo.br/pdf/rcefac/v12n5/44-08.pdf

6. Eberhard L., Braun S., Wirth A., Schindler HJ, Hellmann D., Giannakopoulos NN. The efect of experimental balancing interferences on masticatory performance. J Oral Rehabili., 2014; 41 (5): 346-52. https://www.ncbi.nlm.nih.gov/ pubmed/24843864

7. Alencar Junior F., Becker A. Evaluation of different occlusal splints and counselling in the management of myofascial pain dysfunction. J Oral Rehabil., 2009; 36 (2); 79-85. DOI: 10.1111/j.1365-2842.2008.01913.x.

8. Lobbezoo F., Visscher CM, Naeije M. Treatment of TMD: think twice! In: Manfredini D, ed. Current concepts on temporomandibular disorders. Berlin: Quintessence Publishing, 2010: 483-486.

9. Lang AM. Botulinum toxin therapy for myofascial pain disorders. Curr Pain Headache Rep., 2002; 6 (5): 355-360. DOI: 10.1007 / s11916002-0076-8 .

10. Mazzetto MO, Rodrigues CA, Magri LV, Melchior MO, Paiva G. Severity of DTM in Relation 
to age, Gender and Electromyographic Analysis. Bras Dental Journal, 2014; 28 (1): 54-58. http://www.scielo.br/scielo.php?script=sci_artte xt\&pid=S0103-64402014000100054

11. Jadad AR, Moore RA, Carroll D., Jenkinson C., Reynolds DJ, Gavaghan DJ, et al. Assessing the quality of reports of randomized clinical trials: is blinding necessary? Control Clin Trials, 1996; 17 (1): 1-12. https://www.ncbi.nlm.nih. gov/pubmed/8721797

12. Wahlund K., Nilsson IM, Larsson B. Treating Temporomandibular Disorders in Adolescents: A Randomized, Controlled, Sequential Comparison of Relaxation Training and Occlusal Appliance Therapy. J Oral Facial Pain Headache, 2015; 29 (1): 41-50. DOI: 10.11607/ofph.1285.

13. Alencar Junior FG, Viana PGS, Zamperini CA, Becker AB. Patient Education and Self-Care for the Management of Jaw Pain upon Awakening: A Randomized Controlled Clinical Trial Comparing the Effectiveness of Adding Pharmacologic Treatment with Cyclobenzaprine or Tizanidine. J Oral Facial Pain Headache, 2014; 28:119128. DOI: $10.11607 /$ ofph.963.

14. Niemela K., Korpela M., Raustia A., Stalo PY, Sipila K. Efficacy of stabilisation splint treatment on temporomandibular disorders. J Oral Rehabil., 2012; 39 (11): 799-804. DOI: 10.1111/j.1365-2842.2012.02335.x.

15. Guarda-Nardini G., Stecco A., Stecco C., Masiero S., Manfredini D. Myofascial Pain of the Jaw Muscles: Comparison of Short-Term Effectiveness of Botulinum Toxin Injections and Fascial Manipulation Technique. Cranio, 2012; 30 (2): 95-102. https://www.ncbi.nlm.nih.gov/ pubmed/22606852

16. Doepel M., Nilner M., Ekberg E., Bell YL. Longterm effectiveness of a prefabricated oral appliance for myofascial pain. J Oral Rehabil., 2012; 39 (4): 252-260. http://doczz.com.br/ doc/1252504/volltext----journal-of-craniomandibular-function

17. Shen YF, Younger J., Goddard G., Mackey S. Randomized Clinical Trial of Acupuncture for
Myofascial Pain of the Jaw Muscles. J Orofac Pain., 2009; 23 (4): 353-359. https://www.ncbi. nlm.nih.gov/pmc/articles/PMC2894813/

18. Shen YF, Goddard G. Acupuncture on Myofascial Pain Patients After Clenching. Pain Pract, 2007; 7 (3): 256-264. DOI: 10.1111/j.15332500.2007.00140.x.

19. Calixtre LB, Moreira RF, Franchini GH, Alburquerque-Sendín F., Oliveira AB. Manual therapy for the management of pain and limited range of motion in subjects with signs and symptoms of temporomandibular disorder: a systematic DOI: 10.1111/joor.12321.

20. Ghurye S., McMillan R. Pain-Related Temporomandibular Disorder - Current Perspectives and Evidence-Based Management. Dent Update, 2015; 42 (6): 533-6, 539-42, 545-6. DOI: 10.12968/denu.2015.42.6.533.

21. Michelotti A., lodice G., Vollaro S., Steeks MH, Farella M. Evaluation of the short-term effectiveness of education versus an occlusal splint for the treatment https://www.ncbi.nlm. nih.gov/pubmed/22207667

22. Dworkin SF, Huggins $\mathrm{KH}$, Wilson L., Mancl L., Turner J., Massoth D. et al. A randomized clinical trial using research diagnostic criteria for temporomandibular disorders - axis II to target clinic cases for a tailored self-care TMD treatment program. J Orofac Pain, 2002; 6 (1): 48-63. https://www.ncbi.nlm.nih.gov/ pubmed/11889659

23. Shedden Mora MC, Weber D., Neff A., Rief W. Biofeedback-based cognitive-behavioral treatment compared with occlusal splint for temporomandibular disorder: a randomized controlled trial. Clin J Pain, 2013; 29 (12): 1.057-65. DOI: 10.1097/AJP.0b013e3182850559.

24. Wahlund K., Nilsson IM, Larsson B. Treating temporomandibular disorders in adolescents: a randomized, controlled, sequential comparison of relaxation training and occlusal appliance therapy. J Oral Facial Pain Headache, 2015; 29 (1): 41-50. DOI: 10.11607/ ofph. 1285. 
25. Ouanounou A., Goldberg M., Haas DA. Pharmacotherapy in Temporomandibular Disorders: A Review. Can Dent Assoc., 2017; 83:7. http:// www.jcda.ca/h7

26. Bal Kucuk B., Tolunay Kaya S., Karagoz Motro P., Oral K. Pharmacotherapeutic agents used in temporomandibular disorders. Oral Dis., 2014; 20 (8): 740-3. DOI: 10.1111/odi.12255.

27. Borgini E., Stecco A., Day JA, Stecco C.: How much time is required to modify a fascial fibrosis? J Bodyw Mov Ther., 2010; 14 (4): 318325. DOI: $10.1016 / j . j b m t .2010 .04 .006$

28. Ekberg E., Vallon D., Nilner M. Occlusal appliance therapy in patients with temporomandibular disorders a double-blind controlled study in a short-term https://www.ncbi.nlm. nih.gov/pubmed/9669465

29. Dao TT, Lavigne GJ. Oral splints: The crutches for temporomandibular disorders and bruxism? Crit Rev Oral Biol Med., 1998; 9 (3): 345-361.

30. Al-Ani MZ, Davies SJ, Gray RJ, Sloan P., Glenny AM. Stabilisation splint therapy for temporomandibular pain dysfunction syndrome. Cochrane Database Syst Rev., 2004; 1: CD002778. DOI: 10.1002/14651858.CD002778.pub2.

31. List T., Axelsson S. Management of TMD: Evidence from systematic reviews and meta-analyses. J Oral Rehabil., 2010; 37 (6): 430-451. DOI: $10.1111 / \mathrm{j} .1365-2842.2010 .02089 . x$.
32. Doepel M., Nilner M., Ekberg E., Bell YL. Longterm effectiveness of a prefabricated oral appliance for myofascial pain. J Oral Rehabil., 2012; 39 (4): 252-260. DOI: 10.1111/j.1365$-2842.2011 .02261 . x$.

33. Nardini LG, Stecco A., Stecco C., Masiero S., Manfredini D. Myofascial Pain of the Jaw Muscles: Comparison of Short-Term Effectiveness of Botulinum Toxin Injections and Fascial Manipulation Technique. Cranio, 2012; 30 (2): 95102. DOI: $10.1179 / \mathrm{crn} .2012 .014$

34. Kam E., Eslick G., Campbell I. An audit of the effectiveness of acupuncture on musculoskeletal pain in primary health care. Acupunct Med., 2002; 20 (1): 35-38. https://www.ncbi.nlm.nih. gov/pubmed/11926604

35. Mann F. Acupuncture analgesia in dentistry. Lancet., 1972; 1 (7756): 898-99. https://www. ncbi.nlm.nih.gov/pubmed/4111845

36. Michelotti A., Farella M., Stellato A., Martina R., De Laat A. Tactile and pain threholds in patients with myofascial pain of the jaw muscles: A casecontrol study. J Orofac Pain, 2008; 22 (2): 13945. DOI: 10.5167 / uzh-12355

Submetido em: 17-8-2017

Aceito em: 25-6-2018 